\title{
Complete Disappearance of Hemichorea- Hemiballism due to Hyperglycemia following Acute Ischemic Stroke
}

\author{
Young-Do Kim Hyun-Ji Cho In-Uk Song Sung-Woo Chung \\ Department of Neurology, Catholic University of Korea, Incheon St. Mary's Hospital, Incheon, Korea
}

\section{Dear Sir,}

Hemichorea-hemiballism (HC-HB) is a hyperkinetic disorder that is characterized by continuous, nonpatterned, proximal, and involuntary movements on one side of the body resulting from involvement of the contralateral basal ganglia, particularly the striatum. HC-HB may be associated with a wide variety of diseases, such as cerebrovascular, inflammatory, and infectious diseases, drugs, metabolic derangement, neurodegenerative disorders, and tumors [1,2]. Nonketotic hyperglycemia has occasionally been associated with various neurological abnormalities, including movement disorders [3]. Recently, a few reports have described patients with nonketotic hyperglycemia who presented with HC-HB [4-6]. The HC-HB caused by nonketotic hyperglycemia often abruptly recovered after hyperglycemia had been successfully controlled, but in rare cases, it could be sustained for a few years [7]. To the best of our knowledge, the disappearance of sustained hyperglycemia-induced $\mathrm{HC}-\mathrm{HB}$ following cerebrovascular disease has not yet been documented. Therefore, we herein describe a patient who had complete resolution of hyperglycemia-induced HCHB following a discrete contralateral striatal infarction.

\section{Case}

A 70-year-old right-handed woman with a history of noninsulin-dependent diabetes suddenly developed involuntary movements of the right arm and leg. At that time, blood glucose levels were 500 $\mathrm{mg} / \mathrm{dl}$. The patient manifested continuous, involuntary movements on the right side of the body. Neurological examination showed severe HC-HB with superimposed dystonic posturing on the right side. The movements could not be suppressed voluntarily but ceased during sleep. Muscle tone and the strength of the upper and lower limbs were normal on both sides. There was no evidence of sensory impairment, and cranial nerves were normal. A laboratory examination showed that the glycosylated hemoglobin Alc level was $7.9 \%$. Urine was negative for ketones, indicating nonketotic hyperglycemia. Serum levels of vasculitis factors, blood cell acanthocytosis, and the electroencephalography were all normal. A brain magnetic resonance imaging (MRI) scan showed an irregular area of high-signal intensity involving the left caudate nucleus and putamen in a T1-weighted image (fig. 1). The patient was diagnosed with nonketotic hyperglycemia-induced $\mathrm{HC}-\mathrm{HB}$, and risperi- done ( $3 \mathrm{mg} /$ day) was administered. Clinical manifestations improved slightly, but the HC-HB did not cease completely until the last visit (about 5 months after development of HC-HB). Six months after the onset of $\mathrm{HC}-\mathrm{HB}$, the patient complained of the abrupt onset of right hemiparesis. A neurological examination showed the presence of weakness in the right limbs (MRC score 4 of 5) without other remarkable neurologic deficits. A diffusionweighted image and a T2-weighted image of the brain MRI revealed a discrete highsignal intensity mainly involving the left putamen (fig. 2). The patient had HC-HB in her right arm and leg for 6 months, but the HC-HB of the right side suddenly disappeared following an acute basal ganglia infarction. The patient was treated with an antiplatelet agent for the acute cerebral infarction. The patient has showed no involuntary movements of the right limbs to date.

\section{Discussion}

Nonketotic hyperglycemia-induced HC-HB associated with particular neuroradiological abnormalities has rarely been reported in patients with diabetes mellitus,

\section{KARGER}

Fax +4161306 1234 E-Mail karger@karger.ch www.karger.com
In-Uk Song, $\mathrm{MD}, \mathrm{PhD}$

Department of Neurology, Incheon St. Mary's Hospital Catholic University of Korea

665 Bupyeong-6-dong, Bupyeong-gu, Incheon 403-720 (Korea)

Tel. +82 32280 5010, E-Mail siuy@ catholic.ac.kr 

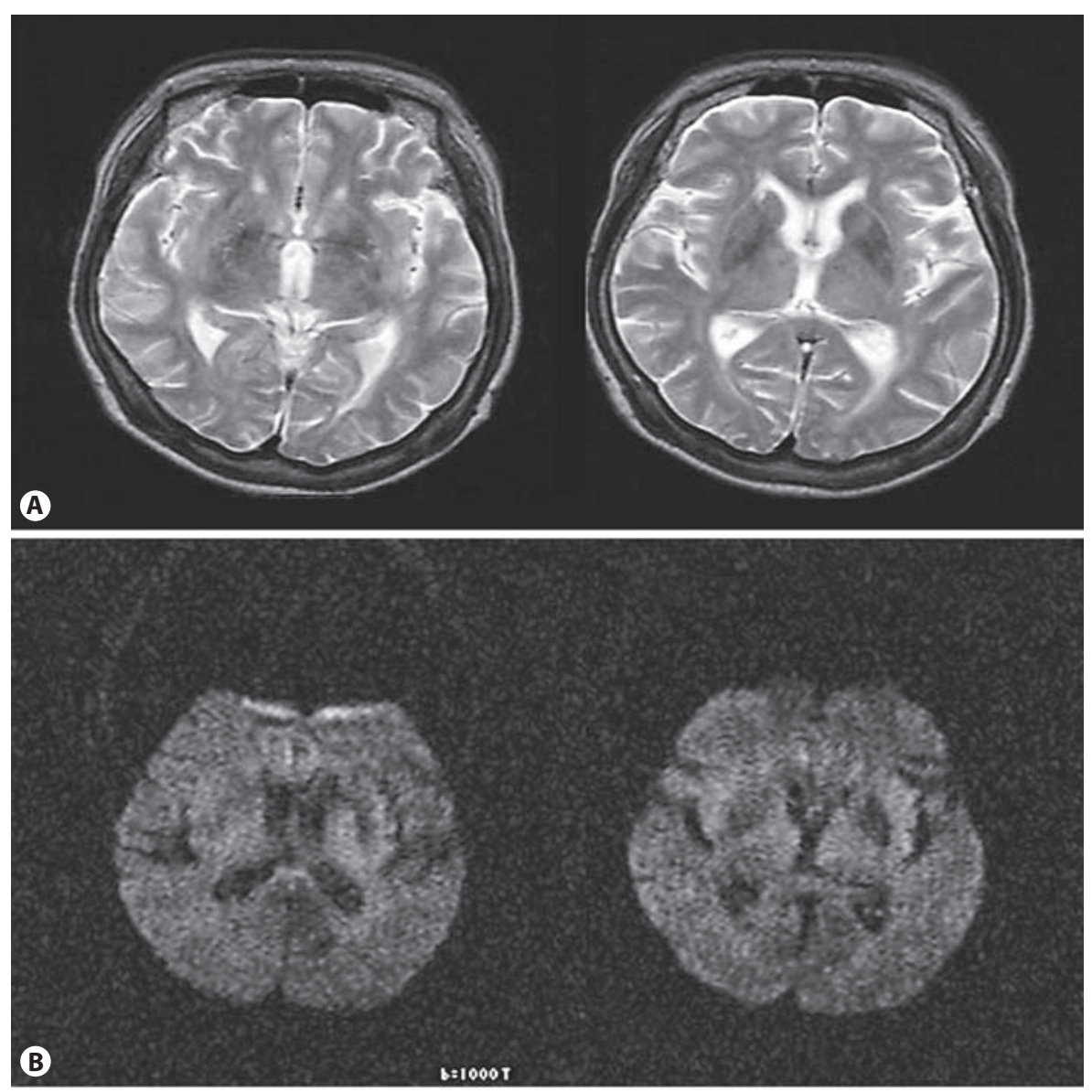

Fig. 1. Magnetic resonance imaging. T2weighted axial images (A) and diffusionweighted images (B) did not show abnormal lesions, but the T1-weighted axial image (C) showed an area of high-signal intensity involving the left caudate nucleus and putamen.

and it usually occurs in elderly women, especially Asian women, with type-2 diabetes, suggesting a genetic influence or an inadequate diabetes control system in underdeveloped countries. The precise mechanism of HC-HB caused by nonketotic hyperglycemia is unknown, although proposed mechanisms for the hyperintense lesions seen in the basal ganglia include postanoxic calcification, petechial hemor-

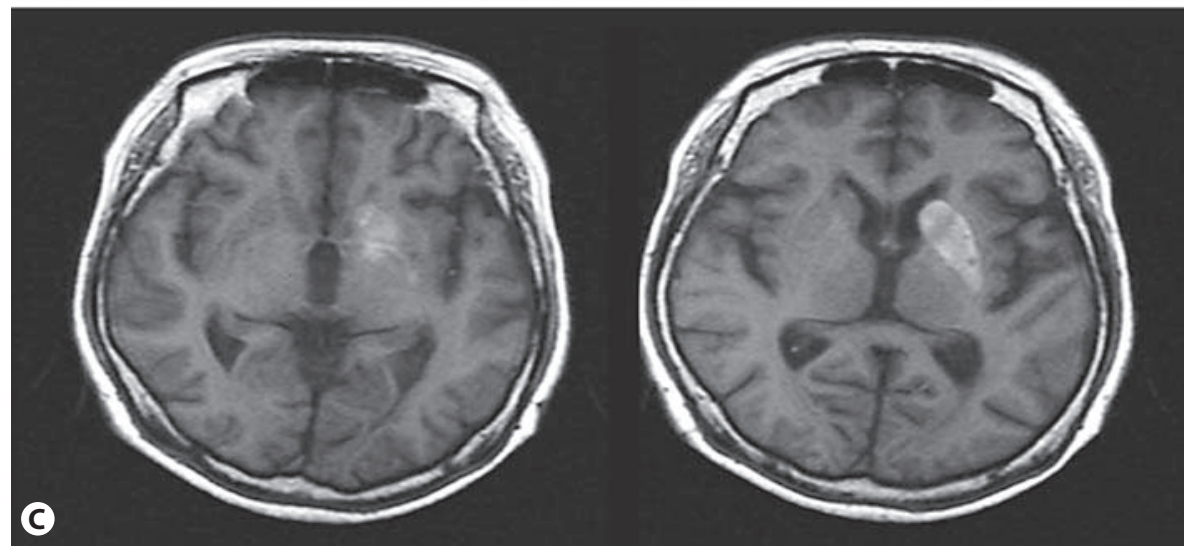

rhage, ischemia, and hyperviscosity with cytotoxic edema $[2,8]$. Common interpretation is based on disinhibition of the deep structures, such as the striatum and thalamus, following lesion of the above-mentioned structures or interruption of their neuronal dopaminergic circuits [9]. However, previous studies have suggested that the pathophysiology of nonketotic hyperglycemia-induced HC-HB is related to met- abolic changes rather than vascular diseases. In particular, a major theory has suggested that decreased $\gamma$-aminobutyric acid (GABA) activity secondary to its metabolism as an alternative energy substrate during a nonketotic hyperglycemic crisis contributes to basal ganglia dysfunction and subsequent chorea. In contrast, GABA can be resynthesized into ketotic hyperglycemia, thus explaining the lack of an associa- 

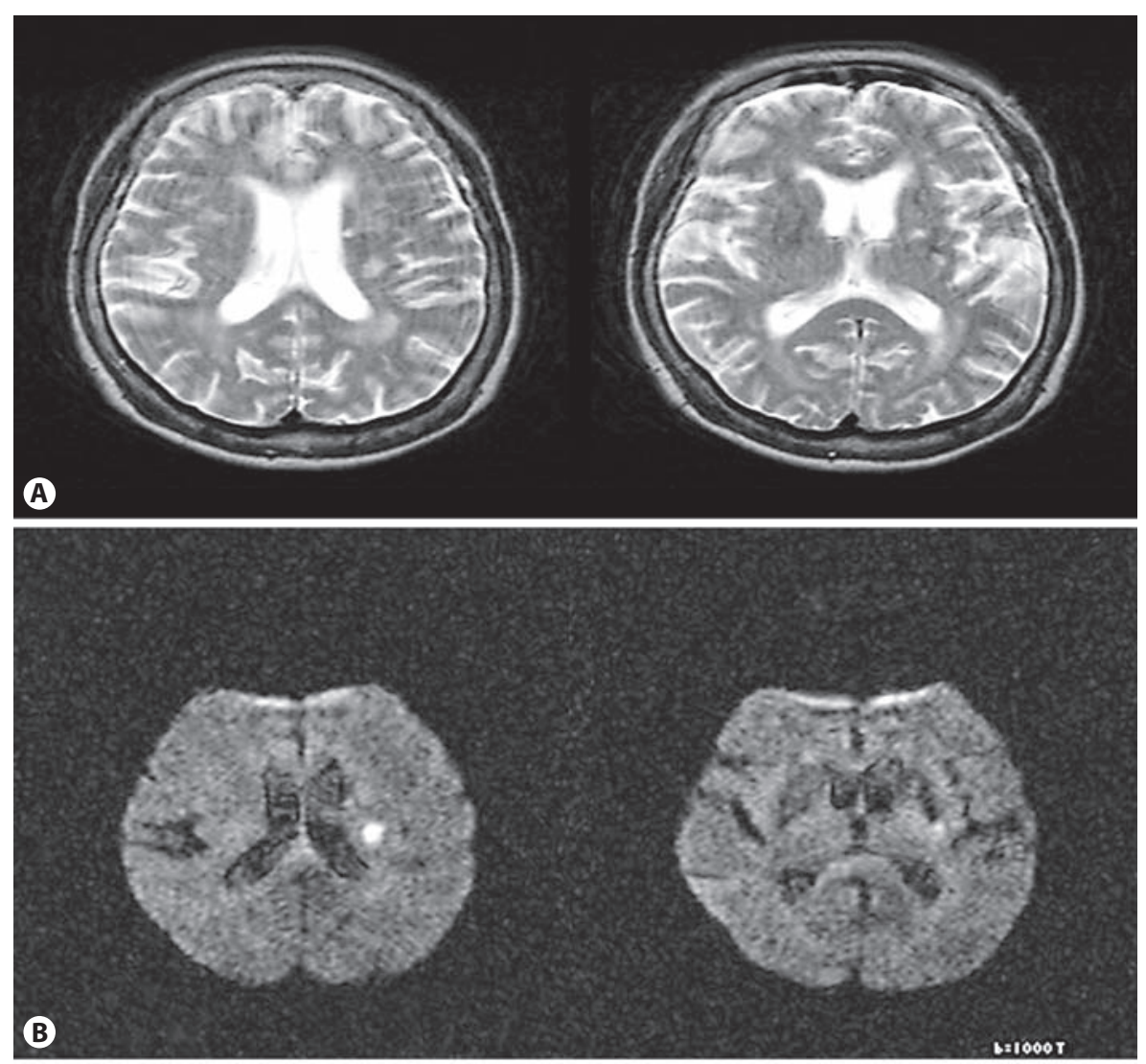

Fig. 2. T2-weighted images (A), apparent diffusion coefficient (B), and diffusionweighted images (C) of the brain MRI showed acute cerebral infarction involving the left striatum.

tion between nonketotic hyperglycemiainduced $\mathrm{HC}-\mathrm{HB}$ and diabetic ketoacidosis [10]. Wang et al. [11] suggested another pathophysiology that chorea may be caused by immunologic diseases, notably the antiphospholipid antibody syndrome, because of an elevation in the IgG level and IgG index on CSF examination [11].

Most patients with nonketotic hyperglycemia-induced HC-HB followed a be-

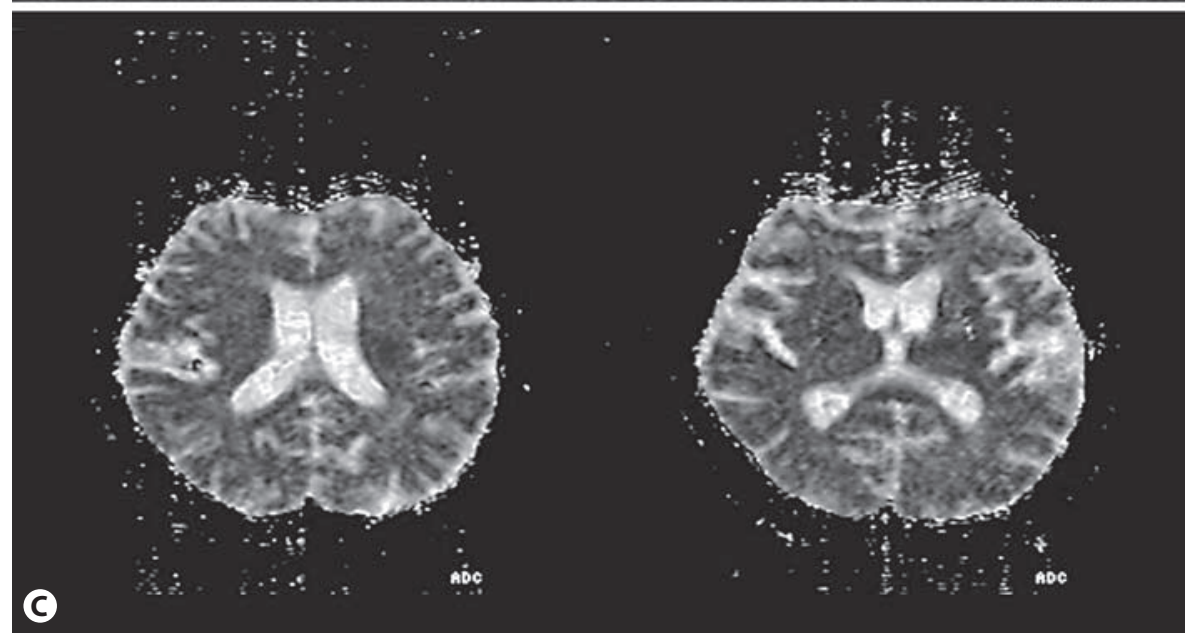

nign course, with complete recovery of the chorea in three quarters of the patients and partial improvement in the remaining quarter [12]. Therefore, most reported patients with chorea or ballism provoked by an episode of nonketotic hyperglycemia recovered from the abnormal involuntary movements within days to a few weeks after normalization of their blood glucose levels [7]. However, unlike previously re- ported cases, the present patient did not show resolution of the $\mathrm{HC}-\mathrm{HB}$ for 6 months until the evolution of left basal ganglia ischemic infarction. Therefore, we assume that, without the acute basal ganglia infarction, the HC-HB would never have disappeared.

Although Le Pira et al. [13] described similar cases, the previous report did not involve the resolution of nonketotic hy- 
perglycemia-induced HC-HB but did involve the disappearance of essential tremor after ischemic stroke. To the best of our knowledge, this report is the first to describe a case with the disappearance of $\mathrm{HC}-\mathrm{HB}$ due to a striatal ischemic infarction. However, the unilateral striatal lesion does not sufficiently explain the phenomenon of the improvement in the nonketotic hyperglycemia-induced HC-HB in this patient.

Hyperkinetic disorders, such as ballismus and chorea, appear to result from the selective involvement of portions of the motor circuit. In the case of ballismus, the disorder results from lesioning or inactivation of the sensorimotor portion of the subthalamic nucleus. Chorea may result from lesions of the putamen, the subthalamic nucleus or, rarely, the thalamus. In addition, both ballismus and chorea are associated with decreased levels of pallidal output. Ablation and deep brain stimulation within the sensorimotor portions of the internal segment of the globus pallidus are highly effective for reducing drug-induced dyskinesias, ballismus, and Huntington's chorea [14]. Therefore, we assume that the $\mathrm{HC}-\mathrm{HB}$ in this case was present due to a striatal lesion caused by nonketotic hyperglycemia and that it disappeared due to a striatal infarction, which resulted in an effect similar to ablation or deep brain stimulation within the globus pallidus or subthalamic nucleus.

In conclusion, we speculate that the striatal ischemic lesion in our patient may have interrupted or reset the hyperactivity of the motor circuit in the basal ganglia caused by nonketotic hyperglycemia and that the consequent alteration in the motor circuit on the opposite side might have manifested as ipsilateral improvement in $\mathrm{HC}-\mathrm{HB}$

\section{References}

- 1 Bellassoued M, Mhiri C, Triki C, Abid M: Hemichorea caused by striatal infarct in a young type 1 diabetic patient. Rev Neurol (Paris) 2001;157:1287-1289.

\2 Chu K, Kang DW, Kim DE, Park SH, Roh JK: Diffusion weighted and gradient echo magnetic resonance findings of hemichorea hemiballismus associated with diabetic hyperglycemia. Arch Neurol 2002;59:448-452.

3 Windebank AJ, McEvoy KM: Diabetes and the nervous system; in Aminoff MJ (ed): Neurology and General Medicine, ed 2. New York, Churchill Livingstone, 1995, pp 349381.

4 Clark JD, Pahwa R, Koller WC, Morales D: Diabetes mellitus presenting as paroxysmal kinesogenic dystonic choreoathetosis. Mov Disord 1995; 10:353-355.

5 Lin JJ, Chang MK. Hemiballism-hemichorea and non-ketotic hyperglycemia. J Neurol Neurosurg Psychiatry 1994;57:748-750.
Lin JJ, Chang MK, Lee CC, Tsao WL: Hemiballism-hemichorea: clinical study in 23 Chinese patients. Zhonghua Yi Xue Za Zhi (Taipei) 1995;55:156-162

7 Ahlskog JE, Nishino H, Evidente VG, Tulloch JW, Forbes GS, Caviness JN, GwinnHardy KA: Persistent chorea triggered by hyperglycaemic crisis in diabetics. Mov Disord 2001;16:890-898.

8 Oh SH, Lee KY, Im JH, Lee MS: Chorea associated with non-ketotic hyperglycemia and hyperintensity basal ganglia lesion on T1-weighted brain MRI study: a meta-analysis of 53 cases including 4 present cases. J Neurol Sci 2002;200:57-62.

$\checkmark 9$ Scoditti U, Rustichelli P, Calzetti S: Spontaneous hemiballism and disappearance of parkinsonism following contralateral lenticular lacunar infarct. Ital J Neurol Sci 1989;10: 575-577.
10 Guisado R, Arieff Al: Neurological manifestations of diabetic comas: correlation with biochemical alterations in the brain. Metabolism 1975;24:665-679.

11 Wang JH, Wu T, Deng BQ, Zhang YW, Zhang P, Wang ZK: Hemichorea-hemiballismus associated with nonketotic hyperglycemia: a possible role of inflammation. J Neurol Sci 2009;284:198-202.

12 Takamatsu K, Ohta T, Sato S, Sano A, Takahashi K, Murakami Y, Yoshihisa K, Sato M, Ohta K: Two diabetics with hemichoreahemiballism and striatal lesions (in Japanese). No To Shinkei 1995;47:167-172.

$>13$ Le Pira F, Giuffrida S, Panetta MR, Lo Bartolo ML, Politi G: Selective disappearance of essential tremor after ischaemic stroke. Eur J Neurol 2004;11:422-423.

14 DeLong, MR, Wichmann T: Circuits and circuit disorders of the basal ganglia. Arch Neurol 2007;64:20-24. 